\title{
Application of Multistage Fuzzy Comprehensive Evaluation and ARMA ( $p$, q) Model to the University Teaching Quality Evaluation System
}

\author{
Baoan $\mathrm{Li}^{1, \mathrm{a}^{*}}$, Peiluan $\mathrm{Li}^{1, \mathrm{~b}}$ and Chunfeng $\mathrm{Liu}^{2, \mathrm{c}}$ \\ ${ }^{1}$ School of Mathematics and Statistics, Henan University of Science and Technology, Luoyang, \\ China \\ ${ }^{2}$ Academic Administration Department, Henan University of Science and Technology, Luoyang, \\ China \\ alibaoan@haust.edu.cn, ${ }^{b}$ Ipllpl_|pl@163.com, ${ }^{\mathrm{c} l i u c h u n f @ h a u s t . e d u . c n}$ \\ * The corresponding author
}

Keywords: University teaching quality; Evaluation system; Analytic hierarchy process; Multistage fuzzy comprehensive evaluation; ARMA $(p, q)$ model

\begin{abstract}
University teaching quality evaluation system is studied. Firs, the mathematical model of university teaching quality evaluation system is established by using analytic hierarchy process and multistage fuzzy comprehensive evaluation, secondly based on the ARMA (p, q) model, the forecast model of university teaching quality evaluation system is established, and the results are the inspected.
\end{abstract}

\section{多级模糊综合评价和ARMA (p, q) 模型在高校教学质 量评价体系中的应用}

\author{
李保安 $^{1, a}$, 李培峦 ${ }^{1, b}$, 刘春峰 ${ }^{2, c}$ \\ 1. 河南科技大学 数学与统计学院, 中国 河南洛阳 471023 \\ 2. 河南科技大学 教务处, 中国 河南 洛阳 471023 \\ alibaoan@ haust.edu.cn, ${ }^{\mathrm{b}}$ lpllp1_lpl@163.com, ${ }^{\mathrm{c}}$ liuchunf@ $@$ haust.edu.cn
}

摘要: 本文研究了高校教学质量评价体系。首先利用层次分析法和模糊综合评价法建立了高 校教学质量评价体系的数学模型, 其次建立基于 $\operatorname{ARMA}(\mathrm{p}, \mathrm{q})$ 模型的高校教学质量评价体系的 预测模型, 并对结果进行了检验。

关键词: 高校教学质量; 评价体系; 层次分析法; 多级模糊综合评价; $\operatorname{ARMA}(p, q)$ 模型

\section{1. 引言}

高校教学质量与学校的生存发展息息相关, 成为了高校时刻向社会不断输送人才的根基 $[1]$ 。 教学质量评价是高校教育评价的核心内容, 是高校保证和提高教学质量的重要措施。因此, 建立客观合理的教学质量评价体系, 是提升教学质量的关键。但是目前我国高校教学质量评 价体系存在一定的问题, 有待改进并加以提高。首先, 教学质量评价标准不规范, 内容过于 死板并缺乏科学性; 其次, 教学评价主体缺乏主动性与参与性 [2]; 最后, 高校对评教结果缺乏 正确的处理方式。

目前我国高校的教学质量评价体系混乱, 评价指标等设计不合理, 评价的方法与设计的模型 也不科学 [3]。本文分别从学生、专家和教师角度来讨论高校教学质量评价体系, 使得研究结 果更加准确和客观, 对于实现高效教学质量评价的科学化和有效化, 全面提高高校教学质量, 具有重要的理论价值和实践意义。 


\section{2. 基于多级模糊综合评价的高校教学质量保障体系的数学模型}

2.1. 高校教学质量评价体系指标的选取

高校教学质量评价体系的评判标准可以分为教学效果、教学方法、教学内容、教学态度与教 学组织。根据对某高校教学质量评价调查数据中无用的、错误的数据进行删除后, 利用 SPSS 软件做上述指标与教学质量评价结果进行相关性分析, 从而得到了各指标对教学质量评价体 系的影响程度, 可以看出教学方法、教学内容、教学态度、教学组织与教学质量评价结果均 有显著性影响。说明我们选取的影响教学质量评价体系的指标比较合适, 具有一定的客观性 与科学性。

2.2. 高校教学质量评价体系的建立

下面将高校教学质量评价体系分为四级。第一级目标层为高校教学质量评价体系; 对于第二 级指标, 考虑到不同学科和不同课程的差异性以及关于教师自评方面, 我们将其分为 $A_{1}$ 学生 评价、 $A_{2}$ 专家评价与 $A_{3}$ 教师评价三个部分; 第三级指标分为 $B_{1}$ 教学效果、 $B_{2}$ 教学内容、 $B_{3}$ 教 学方法、 $B_{4}$ 教学态度、 $B_{5}$ 教学组织; 第四级指标是则对第三级指标的进一步细化 $[4]$ 。具体 如下: $B_{1}$ 教学效果包括: $B_{11}$ 表示达到了教学的预期结果; $B_{12}$ 表示学生的测试成绩得到提高; $B_{13}$ 表示学生的领悟能力提高; $B_{14}$ 表课堂氛围活跃积极。 $B_{2}$ 教学内容包括: $B_{21}$ 表示各方面均 严格要求学生; $B_{22}$ 表示注重开发学生脑力, 启发思维; $B_{23}$ 表示教学方法合理并适合; $B_{24}$ 表 示因材施教, 适合不同水平的学生。 $B_{3}$ 教学方法包括: $B_{31}$ 表示按照教学大纲进行; $B_{32}$ 表示 重难点突出, 详略得当; $B_{33}$ 表示内容准确, 条理清晰。 $B_{4}$ 教学态度包括: $B_{41}$ 表示教学有耐 心, 工作勤奋; $B_{42}$ 表示态度积极乐观; $B_{43}$ 表示认真负责。 $B_{5}$ 教学组织包括: $B_{51}$ 表示教学模 式新颖, 具有创新性; $B_{52}$ 表示上课内容具有对学生有启发性; $B_{53}$ 表示上课的板书工整简洁, 井然有序。

\section{3. 评价模型的建立和求解}

通过对某高校进行问卷调查, 整理相关数据, 综合专家对不同因素的两两比较与其重要性, 我们建立了以下判断矩阵。结果如下:

第一级因素对目标层的判断矩阵为:

$$
A=\left[\begin{array}{ccc}
1 & \frac{5}{3} & \frac{5}{2} \\
\frac{3}{5} & 1 & \frac{3}{2} \\
\frac{2}{5} & \frac{2}{3} & 1
\end{array}\right]
$$

第二级因素层对第一级因素的判断矩阵为:

$A_{1}=\left[\begin{array}{llllr}1 & 0.75 & 0.375 & 1 & 1.5 \\ 1.33 & 1 & 0.5 & 1.33 & 2 \\ 2.67 & 2 & 1 & 2.67 & 4 \\ 1 & 0.75 & 0.375 & 1 & 1.5 \\ 0.67 & 0.5 & 0.25 & 0.67 & 1\end{array}\right], A_{2}=\left[\begin{array}{lllrc}1 & 1.25 & 0.71 & 2.5 & 2.5 \\ 0.8 & 1 & 0.57 & 2 & 2 \\ 1.4 & 1.75 & 1 & 3.5 & 3.5 \\ 0.4 & 0.5 & 0.29 & 1 & 1 \\ 0.4 & 0.5 & 0.29 & 1 & 1\end{array}\right]$,


$A_{3}=\left[\begin{array}{lllll}1 & 1.25 & 1 & 1.25 & 2.5 \\ 0.8 & 1 & 0.8 & 1 & 2 \\ 1 & 1.25 & 1 & 1.25 & 2.5 \\ 0.8 & 1 & 0.8 & 1 & 2 \\ 0.4 & 0.5 & 0.4 & 0.5 & 1\end{array}\right]$ 。

第三级指标对第二级因素层的判断矩阵如下:

$\begin{aligned} B_{1} & =\left[\begin{array}{llll}1 & 0.8 & 0.5 & 1.33 \\ 1.25 & 1 & 0.625 & 1.67 \\ 2 & 1.6 & 1 & 2.67 \\ 0.75 & 0.6 & 0.375 & 1\end{array}\right], B_{2}=\left[\begin{array}{llll}1 & 3 & 6 & 6 \\ 0.33 & 1 & 2 & 2 \\ 0.17 & 0.5 & 1 & 1 \\ 0.17 & 0.5 & 1 & 1\end{array}\right], \\ B_{3} & =\left[\begin{array}{lll}1 & 1.5 & 0.6 \\ 0.67 & 1 & 0.4 \\ 1.67 & 2.5 & 1\end{array}\right], B_{4}=\left[\begin{array}{lll}1 & 2 & 0.5 \\ 0.5 & 1 & 0.5 \\ 1 & 2 & 1\end{array}\right], B_{5}=.\left[\begin{array}{lll}1 & 2.5 & 1.67 \\ 0.4 & 1 & 0.67 \\ 0.6 & 1.5 & 1\end{array}\right] 。\end{aligned}$

然后, 根据上面各个因素层的判断矩阵, 利用 MATLAB 软件分别求出了其对应的最大特征值为 $\lambda_{i}(i=1,2, \cdots, 9)$, 以及 $\lambda_{i}$ 所对应的特征向量 $w_{i}$ 。将特征向量归一化后即为各指标的权重, 结果 如下:

$$
\begin{aligned}
& w_{A}=\left(\begin{array}{lll}
0.5000 & 0.3000 & 0.2000
\end{array}\right) \\
& \lambda_{1}=4.0000 \\
& w_{A_{1}}=\left(\begin{array}{lllll}
0.1500 & 0.1998 & 0.4001 & 0.1500 & 0.1002
\end{array}\right), \lambda_{2}=5.0015 \text {, } \\
& w_{A_{2}}=\left(\begin{array}{lllll}
0.2496 & 0.1999 & 0.34999 & 0.1003 & 0.1003
\end{array}\right), \lambda_{3}=5.0043 \text {, } \\
& w_{A_{3}}=\left(\begin{array}{lllll}
0.2500 & 0.2000 & 0.2500 & 0.2000 & 0.1000
\end{array}\right), \lambda_{4}=5.000, \\
& w_{B_{1}}=\left(\begin{array}{llll}
0.1998 & 0.2501 & 0.4001 & 0.1500
\end{array}\right), \lambda_{5}=4.0002, \\
& w_{B_{2}}=\left(\begin{array}{llll}
0.5997 & 0.1994 & 0.1005 & 0.1005
\end{array}\right), \lambda_{6}=4.0075 \text {, } \\
& w_{B_{3}}=\left(\begin{array}{lll}
0.2998 & 0.2002 & 0.5000
\end{array}\right), \lambda_{7}=3.0023 \text {, } \\
& w_{B_{4}}=\left(\begin{array}{lll}
0.4001 & 0.1999 & 0.3999
\end{array}\right), \lambda_{8}=3.0000 \text {, } \\
& w_{B_{5}}=\left(\begin{array}{lll}
0.5000 & 0.2002 & 0.2998
\end{array}\right), \lambda_{9}=3.0023 \text { 。 }
\end{aligned}
$$

根据公式 $C I=\frac{\lambda_{i}-n}{n-1}$ 和 $C R=\frac{C I}{R I}=\frac{\lambda_{i}-n}{(n-1) R}$ 以及随机一致指标 $R I$ 的数值, 对判断矩阵进行一致 性检验。当 $C R<0.1$ 时, 则表示判断矩阵通过一致性检验。结果如下:

$$
\begin{aligned}
& C R_{A}=\frac{\lambda_{A}-n}{(n-1) R}=0<0.1 \quad C R_{A_{1}}=\frac{\lambda_{A_{1}}-n}{(n-1) R}=\frac{5.0015-5}{4 \times 1.12}=0.00033<0.1, \\
& C R_{A_{2}}=\frac{\lambda_{A_{2}}-n}{(n-1) R}=\frac{5.0043-5}{4 \times 1.12}=0.00096<0.1 \quad C R_{A_{3}}=\frac{\lambda_{A_{3}}-n}{(n-1) R}=0<0.1, \\
& C R_{B_{1}}=\frac{\lambda_{B_{1}} n}{(n-1) R}=\frac{4.0002-4}{3 \times 0.90} \approx 0.000074<0.1, \\
& C R_{B_{2}}=\frac{\lambda_{B_{2}}-n}{(n-1) R}=\frac{4.0075-4}{3 \times 0.90}=0.0028<0.1,
\end{aligned}
$$




$$
\begin{aligned}
& C R_{B_{3}}=\frac{\lambda_{B_{3}}-n}{(n-1) R}=\frac{3.0023-3}{2 \times 0.58}=0.0020<0.1 \\
& C R_{B_{4}}=\frac{\lambda_{B_{4}} n}{(n-1) R}=0<0.1 \quad C R_{B_{5}}=\frac{\lambda_{B_{5}}-n}{(n-1) R}=\frac{3.0023-3}{2 \times 0.58}=0.0020<0.1
\end{aligned}
$$

结果表明以上各个判断矩阵均满足一致性检验的条件。

下面, 我们利用公式 $W_{A}=w_{A}{ }^{T} w_{A_{1}}+w_{A}{ }^{T} w_{A_{2}}+w_{A}{ }^{T} w_{A_{3}}$ 即可求出第二级因素层对目标层的最终的 权重。结果如下:

$$
\begin{aligned}
& w_{B_{1}}=0.1500 \times 0.5+0.2496 \times 0.3+0.25 \times 0.2=0.19988, \\
& w_{B_{2}}=0.1998 \times 0.5+0.1999 \times 0.3+0.2000 \times 0.2=0.19987 。 \\
& \text { 同理可得出, } w_{B_{3}}=0.355047, \quad w_{B_{4}}=0.14509, \quad w_{B_{5}}=0.10019
\end{aligned}
$$

表 1 二级指标的最终权重

\begin{tabular}{lllll}
\hline & $A_{1}$ & $A_{2}$ & $A_{3}$ & $A$ \\
\hline$w_{B_{1}}$ & 0.1500 & 0.2496 & 0.2500 & 0.19988 \\
$w_{B_{2}}$ & 0.1998 & 0.1999 & 0.2000 & 0.19988 \\
$w_{B_{3}}$ & 0.4001 & 0.3499 & 0.2500 & 0.35504 \\
$w_{B_{4}}$ & 0.1500 & 0.1003 & 0.2000 & 0.14509 \\
$w_{B_{5}}$ & 0.1002 & 0.1003 & 0.1000 & 0.10019 \\
\hline
\end{tabular}

即第二级因素层对目标层的最终权重为:

$$
W_{A}=\left(\begin{array}{lllll}
0.1999 & 0.1999 & 0.3550 & 0.1451 & 0.1002
\end{array}\right) 。
$$

该结果表明, 在第三指标中教学效果对教学质量评价体系影响最大, 其次是教学方法与教学 内容, 同时, 在教学态度和教学组织上有待加强。教学方法中最重要的指标是使学生的领悟能 力提高, 教学方法与教学内容方面最重要的指标分别是教学方法合理适合、教学内容按照教 学大纲进行与内容准确并条理清晰。

接下来, 我们需要根据上述层次分析法确定各层评价指标的权重, 然后通过模糊综合评判法 来确定高校教学质量评价体系的最终评价结果。

首先, 根据相关教学管理部门专家确定了高校教学质量评价的等级标准, 即

\section{表 2 高校教学质量评价等级标准}

\begin{tabular}{llllll}
\hline 等级 & 优秀 & 良好 & 中等 & 一般 & 较差 \\
\hline 指标 & $>90$ & $80-90$ & $70-80$ & $60-70$ & $<60$ \\
\hline
\end{tabular}

选取评价集为: $P=\left\{P_{1}, P_{2}, P_{3}, P_{4}, P_{5}\right\}=\{$ 优秀, 良好, 中等, 一般, 较差 $\}$ 。

然后, 根据对该高校教学质量评价体系抽样调查的结果, 我们依次对学生、领导和教师的评 语集进行归一化处理。下面以学生评语集为例 (领导与教师的评价集数据结果略去), 计算出 学生对教师的评价结果, 之后再结合领导与教师的评价即可得出该教师的教学质量水平。结 果如下: 
表 3 高校教学质量评价中学生评语集数据

\begin{tabular}{|c|c|c|c|c|c|c|c|c|c|}
\hline \multirow{19}{*}{$\begin{array}{l}\text { 教 } \\
\text { 学 } \\
\text { 质 } \\
\text { 量 } \\
\text { 评 } \\
\text { 价 } \\
\text { 结 } \\
\text { 果 }\end{array}$} & \multicolumn{2}{|l|}{ 一级指标 } & \multicolumn{2}{|c|}{ 二级指标 } & \multicolumn{5}{|c|}{ 学生评语集 } \\
\hline & 内容 & 权重 & 内容 & 权重 & 优秀 & 良好 & 较好 & 一般 & 较差 \\
\hline & & & $B_{11}$ & 0.1998 & 0.75 & 0.15 & 0.1 & 0 & 0 \\
\hline & 奴览效田 & مחת 10 & $B_{12}$ & 0.2501 & 0.7 & 0.2 & 0.1 & 0 & 0 \\
\hline & 双子双木 & 0.1999 & $B_{13}$ & 0.4001 & 0.65 & 0.15 & 0.1 & 0.1 & 0 \\
\hline & & & $B_{14}$ & 0.15 & 0.5 & 0.4 & 0.05 & 0 & 0.05 \\
\hline & & & $B_{21}$ & 0.5997 & 0.5 & 0.35 & 0.15 & 0 & 0 \\
\hline & 教。 & o 100 & $B_{22}$ & 0.1994 & 0.75 & 0.15 & 0.05 & 0.05 & 0 \\
\hline & 抎 & 0.1999 & $B_{23}$ & 0.1005 & 0.5 & 0.5 & 0 & 0 & 0 \\
\hline & & & $B_{24}$ & 0.1005 & 0.45 & 0.25 & 0.1 & 0.1 & 0.1 \\
\hline & & & $B_{31}$ & 0.2998 & 0.6 & 0.1 & 0.15 & 0.1 & 0.05 \\
\hline & 教学内容 & 0.35504 & $B_{32}$ & 0.2002 & 0.5 & 0.2 & 0.1 & 0.2 & 0 \\
\hline & & & $B_{33}$ & 0.5 & 0.55 & 0.25 & 0.1 & 0 & 0.1 \\
\hline & & & $B_{41}$ & 0.4001 & 0.4 & 0.5 & 0.1 & 0 & 0 \\
\hline & 教学态度 & 0.1451 & $B_{42}$ & 0.1999 & 0.6 & 0.3 & 0.1 & 0 & 0 \\
\hline & & & $B_{43}$ & 0.3999 & 0.65 & 0.25 & 0 & 0.1 & 0 \\
\hline & & & $B_{51}$ & 0.5 & 0.5 & 0.2 & 0.2 & 0.1 & 0 \\
\hline & 教学组织 & 0.1002 & $B_{52}$ & 0.2002 & 0.7 & 0.15 & 0.15 & 0 & 0 \\
\hline & & & $B_{53}$ & 0.2998 & 0.6 & 0.1 & 0.15 & 0.1 & 0.05 \\
\hline
\end{tabular}

根据表 3 的结果可得出，从三级指标到学生评语集的模糊矩阵为:

$$
\begin{aligned}
& R_{1}=\left[\begin{array}{lllll}
0.75 & 0.15 & 0.1 & 0 & 0 \\
0.7 & 0.2 & 0.1 & 0 & 0 \\
0.65 & 0.15 & 0.1 & 0.1 & 0 \\
0.5 & 0.4 & 0.05 & 0 & 0.05
\end{array}\right], \quad R_{2}=\left[\begin{array}{lllll}
0.5 & 0.35 & 0.15 & 0 & 0 \\
0.75 & 0.15 & 0.05 & 0.05 & 0 \\
0.5 & 0.5 & 0 & 0 & 0 \\
0.45 & 0.25 & 0.1 & 0.1 & 0.1
\end{array}\right] \text {, } \\
& R_{3}=\left[\begin{array}{lllll}
0.6 & 0.1 & 0.15 & 0.1 & 0.05 \\
0.5 & 0.2 & 0.1 & 0.2 & 0 \\
0.55 & 0.25 & 0.1 & 0 & 0.1
\end{array}\right], \quad R_{4}=\left[\begin{array}{lllll}
0.4 & 0.5 & 0.1 & 0 & 0 \\
0.6 & 0.3 & 0.1 & 0 & 0 \\
0.65 & 0.25 & 0 & 0.1 & 0
\end{array}\right] \\
& R_{5}=\left[\begin{array}{lllll}
0.5 & 0.2 & 0.2 & 0.1 & 0 \\
0.7 & 0.15 & 0.15 & 0 & 0 \\
0.6 & 0.1 & 0.15 & 0.1 & 0.05
\end{array}\right]
\end{aligned}
$$

通过层次分析法所求的第二级指标 $B_{i}(i=1,2, \cdots, 5)$ 对于目标层的最终权重

$$
W_{A}=\left(\begin{array}{lllll}
0.1999 & 0.1999 & 0.35504 & 0.1451 & 0.1002
\end{array}\right) \text {, }
$$

从而可以得出第三级指标的各个评判矩阵 $B_{i}(i=1,2, \cdots, 5)$, 即

$$
\begin{aligned}
& B_{1}=w_{B_{1}} R_{1}=\left[\begin{array}{lllll}
0.6600 & 0.2000 & 0.0925 & 0.0400 & 0.0075
\end{array}\right], \\
& B_{2}=w_{B_{2}} R_{2}=\left[\begin{array}{lllll}
0.5450 & 0.3153 & 0.1100 & 0.0200 & 0.0101
\end{array}\right], \\
& B_{3}=w_{B_{3}} R_{3}=\left[\begin{array}{lllll}
0.555 & 0.1950 & 0.1150 & 0.0700 & 0.0065
\end{array}\right],
\end{aligned}
$$




$$
\begin{aligned}
& B_{4}=w_{B_{4}} R_{4}=\left[\begin{array}{lllll}
0.5399 & 0.3600 & 0.0600 & 0.0400 & 0
\end{array}\right], \\
& B_{5}=w_{B_{5}} R_{5}=\left[\begin{array}{lllll}
0.5700 & 0.1600 & 0.1750 & 0.0800 & 0.0150
\end{array}\right] \text { 。 }
\end{aligned}
$$

那么第三级指标的综合评判矩阵为:

$$
R_{1}=\left[\begin{array}{l}
B_{1} \\
B_{2} \\
B_{3} \\
B_{4} \\
B_{5}
\end{array}\right]=\left[\begin{array}{lllll}
0.6600 & 0.2000 & 0.0925 & 0.0400 & 0.0075 \\
0.5450 & 0.3153 & 0.1100 & 0.0200 & 0.0101 \\
0.5555 & 0.1950 & 0.1150 & 0.0700 & 0.0650 \\
0.5399 & 0.3600 & 0.0600 & 0.0400 & 0 \\
0.5700 & 0.1600 & 0.1750 & 0.0800 & 0.0150
\end{array}\right] \text {, }
$$


假定评价集合组成的向量 $M=\left[\begin{array}{lllll}95 & 85 & 75 & 65 & 55\end{array}\right]^{T}$, 则学生的评价结果为: $L_{1}=B M=87.8190$ ，表明学生对教师的教学质量评价结果为良好。

同理, 按照上述求解学生对教师的教学质量评价结果的方法, 可以分别得到领导与教师对教 学质量评价的结果。

对于领导因素而言, 综合评判矩阵为:

$R_{2}=\left[\begin{array}{lllll}0.5325 & 0.1950 & 0.1275 & 0.1050 & 0.0400 \\ 0.5751 & 0.3249 & 0.0550 & 0.0050 & 0.0400 \\ 0.4350 & 0.2950 & 0.1000 & 0.0700 & 0.1000 \\ 0.4999 & 0.2400 & 0.1500 & 0.0700 & 0.0400 \\ 0.4700 & 0.1700 & 0.1500 & 0.1600 & 0.0500\end{array}\right]$,

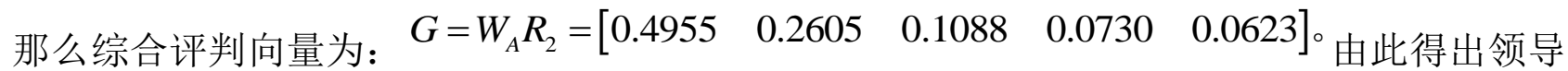
的结果为: $L_{2}=G M=85.5465$ ，上述结果也表明领导对教师的评价结果为良好。 对于教师因素而言, 综合评判矩阵为:

$R_{3}=\left[\begin{array}{lllll}0.5975 & 0.1875 & 0.0800 & 0.0700 & 0.0650 \\ 0.4451 & 0.2700 & 0.1800 & 0.0900 & 0.0151 \\ 0.4100 & 0.2601 & 0.1900 & 0.0700 & 0.0700 \\ 0.5099 & 0.3300 & 0.1200 & 0.0400 & 0 \\ 0.4700 & 0.1250 & 0.1800 & 0.1601 & 0.0650\end{array}\right]$,

则 $U=W_{A} R_{3}=\left[\begin{array}{lllll}0.4750 & 0.2442 & 0.1549 & 0.0787 & 0.0474\end{array}\right]$ 。那么领导对教师的综合评价结果 为: $L_{3}=W_{A} U=85.2220$, 表明教师对教学质量评价的结果也为良好。

根据第一级因素层 (即学生、领导与教师) 对高校教学质量评价体系的影响我们所求出的各 自权重为: $w_{A}=\left(\begin{array}{lll}0.5000 & 0.3000 & 0.2000\end{array}\right)$, 及上面所求的学生、领导与教师对高校教学质 量的评价结果, 令 $Y=\left[\begin{array}{lll}87.8190 & 85.5465 & 85.2220\end{array}\right]^{T}$, 那么最后的评价结果为: $Q=w_{A} \cdot Y \approx 86.6179$, 即高校的教学质量评价体系整体表现为良好。

\section{3. $\operatorname{ARMA}(p, q)$ 预测模型}

\section{1 ARMA $(p, q)$ 模型}


根据高校教学质量评价的数据, 首先判断该数据是否为平稳型序列; 然后再按照 ARMA (p, q) 模型的计算过程，可以计算出高校教学质量评价体系结果趋势。

根据搜集的近几年高校教学质量评价数据, 定性的判断出高校教学质量评价体系的走向, 如 下图所示:

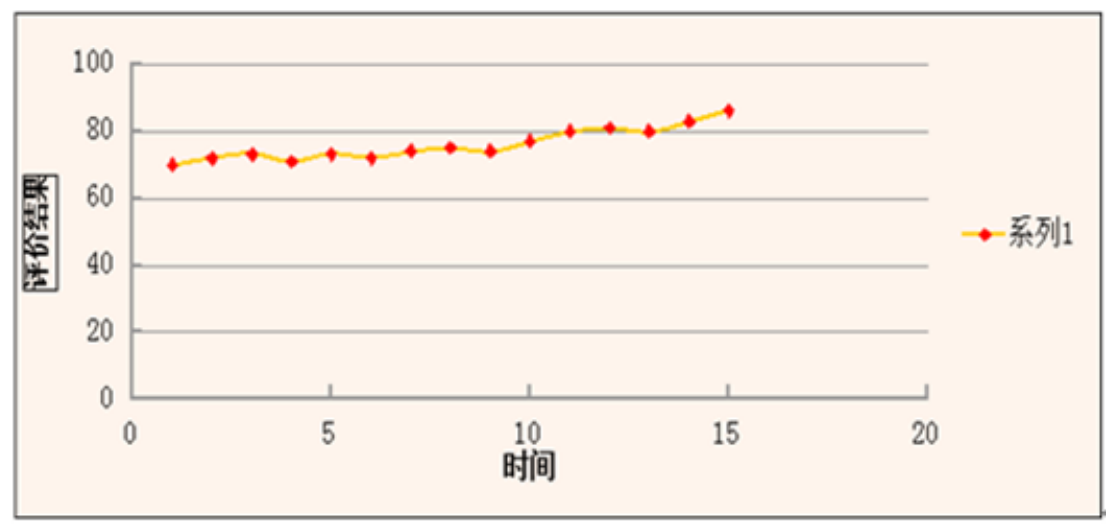

图 1 近十年高校教学质量评价结果趋势图

从图 1 可以看到高校教学质量评价体系结果呈上升趋势, 教学质量水平一直在提高。从整体 来看, 高校教学质量评价体系结果大致具有一次线性关系, 逐渐增加并不具有平稳性, 因此 我们需要对其进行一阶差分运算, 结果如下:

表 4 评价结果值与一阶差分值

\begin{tabular}{|c|c|c|c|c|c|}
\hline 时间 & $\begin{array}{l}\text { 评 价结 } \\
\text { 果 }\end{array}$ & $\begin{array}{l}\text { 一阶差 } \\
\text { 分 }\end{array}$ & 时间 & $\begin{array}{l}\text { 评价结 } \\
\text { 果 }\end{array}$ & $\begin{array}{l}\text { 一阶差 } \\
\text { 分 }\end{array}$ \\
\hline 1 & 70 & & 9 & 74 & -1 \\
\hline 2 & 72 & 2 & 10 & 77 & 3 \\
\hline 3 & 73 & 1 & 11 & 80 & 3 \\
\hline 4 & 71 & -2 & 12 & 81 & 1 \\
\hline 5 & 73 & 2 & 13 & 80 & -1 \\
\hline 6 & 72 & -1 & 14 & 83 & 2 \\
\hline 7 & 74 & 2 & 15 & 86 & 3 \\
\hline 8 & 75 & 1 & & & \\
\hline
\end{tabular}

对原高校教学质量评价体系结果进行一阶差分后, 根据差分数据绘制出了一阶差分后的时间 序列图，如下: 


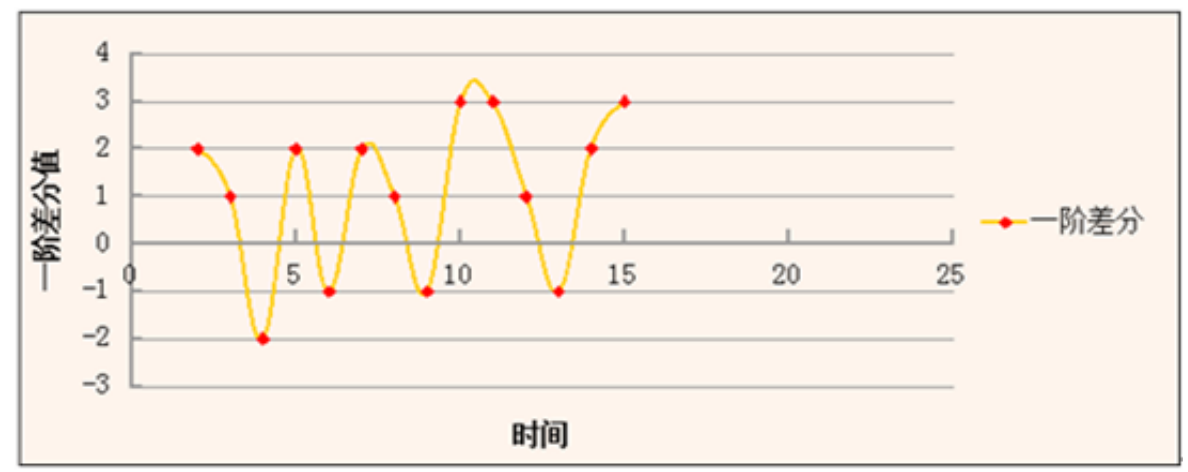

图 2 一阶差分时间序列图

从图 2 可以看出, 一阶差分后的高校教学质量评价体系结果在零值附近来回波动, 波动范围 比较小，具有了很强的稳定性。

那么下面就可以对其进行模型的识别阶段, 以此来检测所选模型的合理性。即分别求出高校 教学质量评价结果组成的时间序列的自相关系数和偏自相关系数, 确定自相关阶数 $\mathrm{p}$ 和移动 平均阶数 q。ARMA (p, q) 模型定阶原则如下所示:

表 $5 \operatorname{ARMA}(\mathrm{p}, \mathrm{q})$ 模型定阶表

\begin{tabular}{lll}
\hline $\mathrm{ACF}$ & $\mathrm{PACF}$ & 模型定阶 \\
\hline 拖尾 & $\mathrm{p}$ 阶截尾 & $\mathrm{AR}(\mathrm{p})$ 模型 \\
$\mathrm{q}$ 阶截尾 & 拖尾 & $\mathrm{MA}(\mathrm{q})$ 模型 \\
拖尾 & ARMA $(\mathrm{p}, \mathrm{q})$ 模 \\
& 拖尾 & 型 \\
\hline
\end{tabular}

对高校教学质量评价体系一阶差分数据进行处理后, 利用 SPSS 软件可以得出高校教学质量评 价结果的自相关系数与偏自相关系数, 以及一阶差分时间序列自相关分析图和偏自相关图。 可以看出自相关系数与偏自相关系数从 $t=2$ 开始就逐渐衰减, 并逐渐与零值靠近。可以初步 判定此时间序列的自相关阶数 $p=2$ 和移动平均阶数 $q=2$ 。

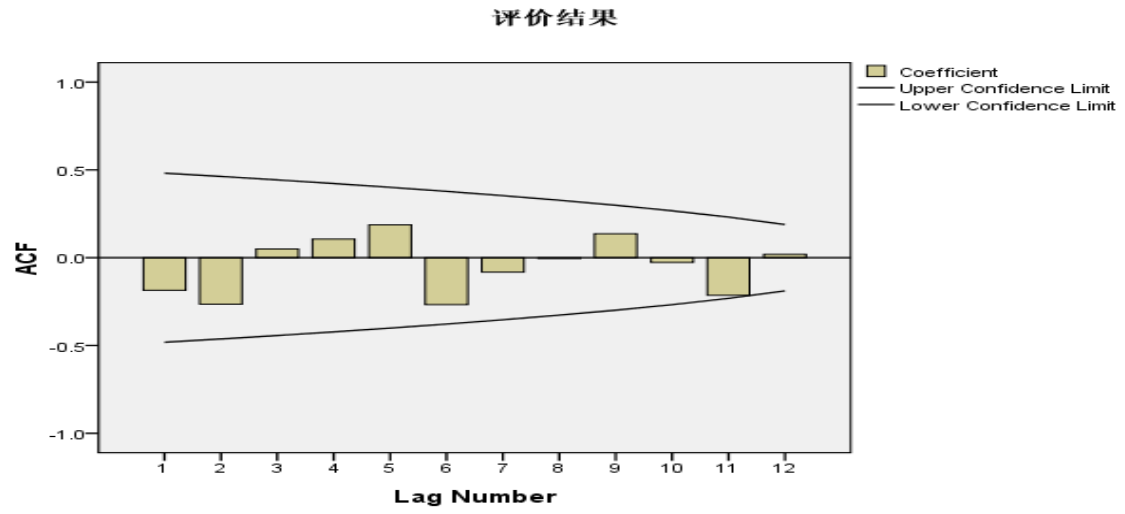

图 3 一阶差分自相关函数分析图 


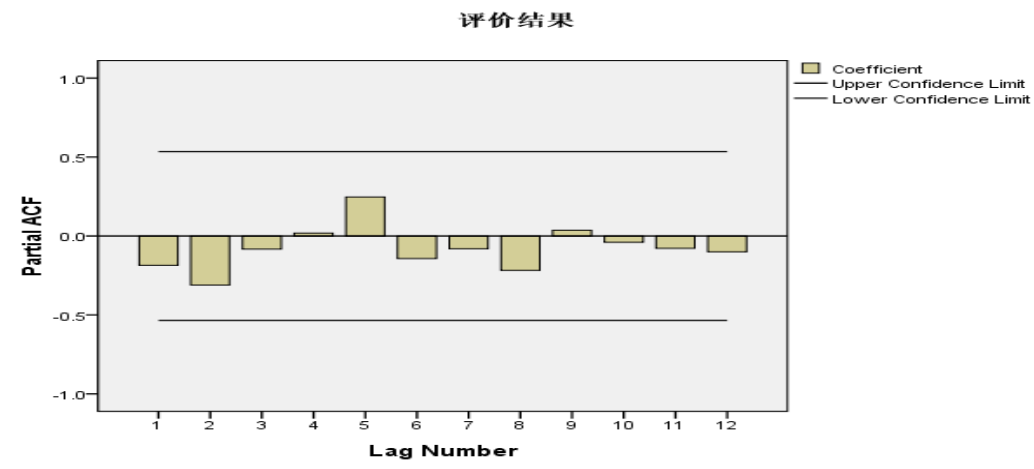

图 4 一阶差分偏自相关函数分析图

从一阶差分的自相关函数与偏自相关函数的图像得出, 两者均表现为明显的拖尾性质, 那么 就得到高校教学质量评价结果该时间序列适合 ARMA (p, q) 中的 ARIMA (p, d, q) 模型, 其中 $d$ 表 示差分阶数。

综合以上，那么高校教学质量评价结果组成的时间序列适合 ARIMA $(2,1,2)$ 模型。并得出了模 型参数估计值为:

表 6 ARIMA $(2,1,2)$ 模型参数估计结果

ARIMA Model Parameters

\begin{tabular}{|c|c|c|c|c|c|c|c|c|}
\hline & & & & & Estimate & $\mathrm{SE}$ & $t$ & Sig. \\
\hline \multirow[t]{7}{*}{ 评价结果-槙型_1 } & \multirow[t]{6}{*}{ 评价结果 } & \multirow[t]{6}{*}{ No Transformation } & \multicolumn{2}{|l|}{ Constant } & -.121 & .314 & -385 & .710 \\
\hline & & & AR & Lag 1 & -151 & .898 & -168 & .870 \\
\hline & & & & Lag 2 & -.451 & .349 & -1.293 & .232 \\
\hline & & & Difference & & 1 & & & \\
\hline & & & MA & Lag 1 & .970 & 41.279 & .023 & .982 \\
\hline & & & & Lag 2 & .027 & 2.142 & .013 & .990 \\
\hline & 时间 & No Transformation & Numerator & $\operatorname{Lag} 0$ & .132 & .035 & 3.730 & .006 \\
\hline
\end{tabular}

由上表可得出模型式为: $y_{t}=-0.121-0.151 y_{t-1}-0.451 y_{t-2}+0.97 \varepsilon_{t-1}+0.27 \varepsilon_{t-2}+\varepsilon_{t}$ 。 3.2. 模型的检验

为判断以上建立模型的优劣, 需对该模型进行残差序列检验。若该时间序列为白噪声序列, 表明通过检验, 所设模型合理, 可以进行下面高校教学质量评价结果的预测; 反之, 若检验 不通过, 则需对模型进行调整, 重设 $(p, q)$ 值, 并反复试验直至通过检验。利用 SPSS 软件得 出的残差序列自相关与偏自相关图如下: 


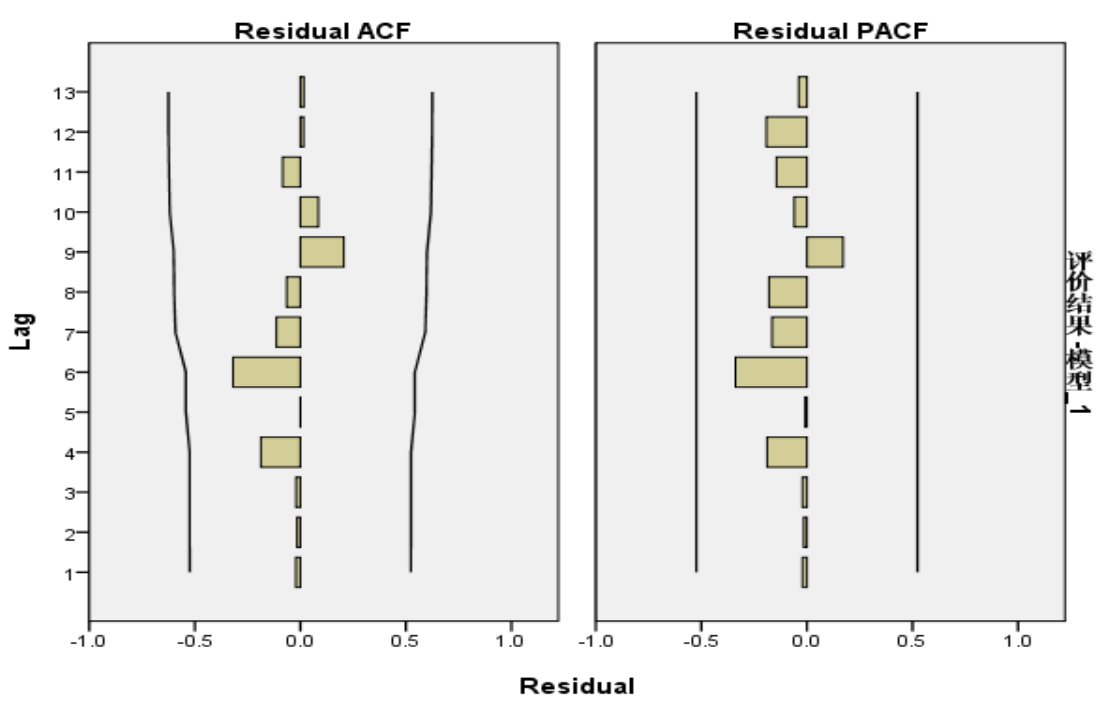

图 5 残差分析图

从图中可以看出, 数值均在零值附近波动, 与零非常的逼近。因此, 可以判断出该时间序列 式白噪声序列, 则以上模型通过了检验。

2.3. 模型的预测

模型通过检验后, 那么就可以利用 ARIMA $(2,1,2)$ 对未来高校教学质量评价体系结果进行预测。 下面以预测未来 5 年为例, 运行结果如下:

表 7 ARIMA $(2,1,2)$ 模型预测结果

Forecast

\begin{tabular}{|ll|r|r|r|r|r|}
\hline Model & & \multicolumn{1}{|c|}{16} & \multicolumn{1}{|c|}{17} & \multicolumn{1}{c|}{18} & \multicolumn{1}{c|}{19} & \multicolumn{1}{c|}{20} \\
\hline 评价结果-模型_1 & Forecast & 87 & 89 & 92 & 94 & 96 \\
& UCL & 90 & 92 & 95 & 97 & 100 \\
& LCL & 84 & 86 & 88 & 91 & 93 \\
\hline
\end{tabular}

从表 7 可以看出, 在未来 5 年, 我国高校教学质量评价体系结果呈上升趋势。并在 3 年后, 高校教学质量评价水平达到 90 分以上, 评价等级达到优秀。该结果也从侧面反映了我国未来 将加大对高校教学质量评价体系的管理力度。

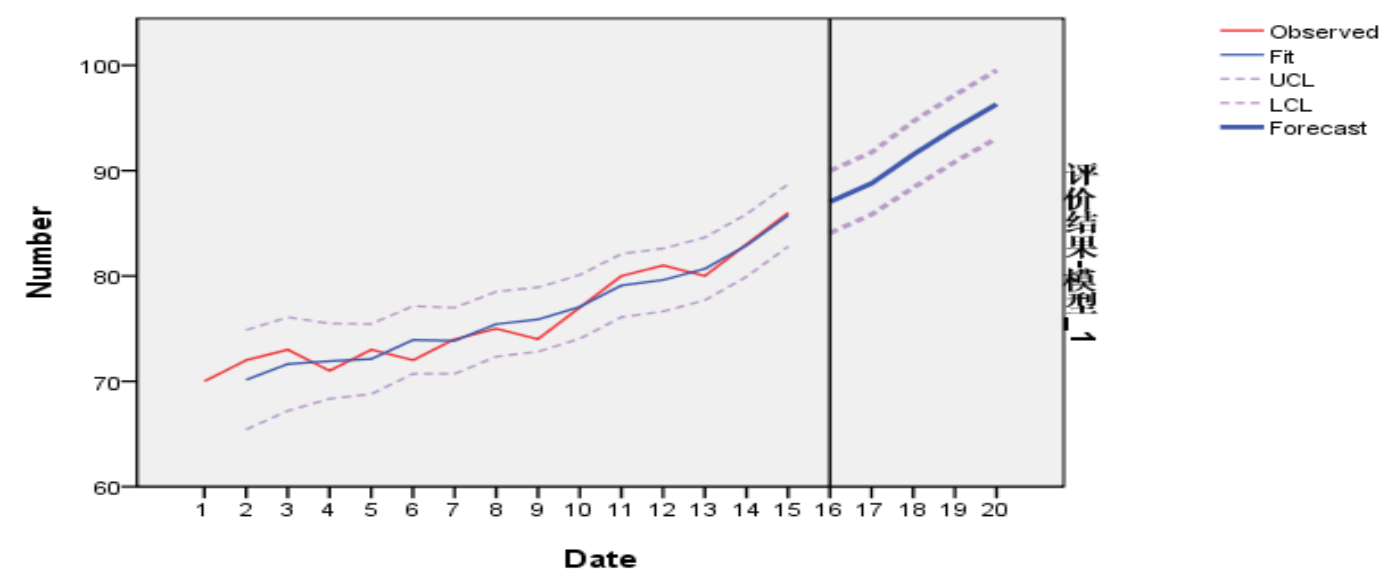

图 6 预测结果

图 6 也表明了该模型拟合的很合理, 同时也清楚地看出了未来我国高校教学质量评价体系结 果有了显著的提升。 


\section{4. 致谢}

基金项目: 国家自然科学基金项目 (11101126，11261010), 河南省高等教育教学改革研究项 目 (2014SJG LX195)，河南科技大学重大教育教学改革研究项目 (2014ZD-007)。

\section{参考文献}

[1] 宋珂. 高校教学质量评价[J]. 中国科教创新导刊, 2011（35）：154.

[2] 张典兵. 高校教学质量评价存在的问题与对策 $[J]$. 继续教育研究, 2006 (1) : 115-117.

[3] 宋建尊, 张甫仁, 杨雅怡. 高校教师教学质量评价中存在的问题及对策研究[J]. 科技视界, 2012 (22) : 89-90.

[4] 曾松伟, 吴胜达, 胡海根, 周素茵. 基于层次分析法的高校教学质量评价改革 $[\mathrm{J}]$. 绍兴文 理学院学报, 2012，32(10)：89-94.

[5] 赵春元. 基于层次分析的教学质量模糊综合评价模型及应用 $[\mathrm{J}]$. 沈阳工程学院学报(自然 科学版), 2011,7 (2) : 185-189.

[6] 丁家玲, 叶金华. 层次分析法和模糊综合评判在教师课堂教学质量评价中的应用 [J]. 武汉 大学学报（社会科学版），2003，56（2）：241-245.

[7] 徐向艺. 高等学校教学质量管理的范畴、原则与体系 [J]. 高等理科教育, 2004（1）：40-45.

[8] 陈艳格. 试论高校教学质量评价体系的构建 $[\mathrm{J}]$. 教育与职业, 2012 （23）：185-186.

[9] 李培峦, 李保安, 刘春峰. 基于多级模糊综合评价的高校教学质量保障体系的模型研究[J]. 科技创新导报, 2015（22）：1-2.

[10]姜启源, 谢金星, 叶俊. 数学模型 $[M]$. 第四版. 北京: 高等教育出版社, 2011年.

\section{Acknowledgement}

This research was financially supported by the national natural science foundation of China (11101126, 11261010), the Research Project of Higher Education Reform in Henan (2014SJG LX195) and the Teaching Reform Research Planning Project of Henan University of Science and Technology (2014ZD-007)

\section{References}

[1] Song Ke. Universities teaching quality evaluation. China innovation of science and Education Journal. 2011, No. 35, p. 154-154.

[2] Zhang Dianbing. The problems existing in the university teaching quality evaluation and countermeasures. Continue Education Research. 2006, No. 1, p. 115-117.

[3] Song Jianzun, Zhang Furen, Yang Yayi. Research of University teachers' teaching quality evaluation of the existing problems and countermeasures. Science \& Technology Vision. 2012, No. 22, p. 89-90.

[4] Zeng Songwei, Wu Shengda, Hu Haigen, et al. Reform of University Teachers'Performance Based on Analytic Hierarchy Process. Journal of Shaoxing University. Vol. 32 (2012) No. 10, p. 89-94.

[5] Zhao Chunyuan. Application of fuzzy judgment based on AHP model in the evaluation of teaching quality. Journal of Shenyang Institute of Engineering (Natural Science). Vol. 7 (2011) No. 2, p. 185-189. 
[6] Ding Jialing, Ye Jinhua. On AHP Model and Fuzzy Judgement in Evaluation of Undergraduate Teaching Quality. Journal of Wuhan University of Hydraulic and Electrical Engineering (Social Sciences Edition). Vol. 56 (2003) No. 2, p. 241-245.

[7] Xu Xiangyi. Category, Principle and System of Uinversity teaching quality management. Higher Education of Science. 2004, No. 1, p. 40-45.

[8] Chen Yange. Discussion on the Construction of Universities Teaching Quality Evaluation System. Education and Vocation. 2012, No. 23, p. 185-186.

[9] Li Peiluan, Li Baoan, Liu Chunfeng. Research of Teaching Quality Guarantee System Model for Universities based on the Multistage Fuzzy Comprehensive Evaluation. Science and Technology Innovation Herald. 2015, No. 22, p. 1-2.

[10]Jiang Qiyuan, Xie Jinxing, Ye Jun. Mathematical Model. China Higher Education Press, 2011, p. 249-269.

作者简介: 李保安 (1972-), 男, 河南洛阳, 副教授, 主要研究方向为非线性系统优化与 控制、数学建模, E-mail : libaoan@haust. edu.cn。 